injury may have occurred by gradual erosion as the cup and its cement migrated medially.

Whatever the precise mechanism, treatment depends on early recognition. In our case prolonged urinary drainage by catheter was the only treatment needed; after five weeks drainage from the wound had stopped, the fistulae had all healed, and a repeat cystogram showed no extravasation of contrast medium into the area of the acetabulum.

\section{REFERENCES}

Kumar JM, Jowett RL. Fistula between the hip and caecum. $J$ Bone Joint Surg [Br] 1984:66-B:603.

Lowell JD, Davis JAK, Bennet AH. Bladder fistula following total hip replacement using self-curing acrylic. Clin Orthop 1975:111:131-3.

Reiley MA, Bond D, Branick RI, Wilson EH. Vascular complications following total hip arthroplasty: a review of the literature and a report of two cases. Clin Orthop 1984:186:23-8.

Solomon MH, MacGregor RJ. Uretero-cutaneous fistula following hip surgery. J Urol 1980;124:427-8.

\title{
ANEURYSM AFTER ARTHROSCOPY
}

\author{
M. P. MANNING, J. H. MARSHALL
}

A 23-year-old man twisted his right knee whilst playing association football. When seen seven weeks after the injury, he had a small effusion, slight quadriceps wasting, and tenderness over the medial joint line. There was no obvious instability and radiographs were normal.

Diagnostic arthroscopy was performed under general anaesthesia, with a pneumatic thigh tourniquet. Medial and lateral joint-line stab incisions were made, and he was found to have a complete tear of the anterior cruciate ligament. The wounds were closed with two Prolene skin sutures and a wool-and-crêpe dressing applied before releasing the tourniquet.

At an outpatient clinic two weeks later the dressing (more bloodstained than usual) was removed. The wounds were clean and dry, but beneath the lateral wound was a pulsatile swelling $1.5 \mathrm{~cm}$ in diameter with a bruit over it. The knee was bandaged, but the swelling slowly enlarged and six weeks later he was readmitted. Under general anaesthesia and a pneumatic thigh tourniquet the lateral incision was reopened and slightly enlarged. A false aneurysm was found and excised. The vessel feeding the aneurysm, the inferior lateral geniculate artery, was doubly ligated and the tourniquet was then released to ensure that haemostasis had been secured. The skin was sutured. He made an uneventful recovery but has subsequently needed prosthetic replacement of the anterior cruciate ligament.

Discussion. Arthroscopy has become a commonly performed procedure with few reported complications. In a

M. P. Manning, FRCS, Orthopaedic Registrar

Royal Liverpool Children's Hospital, Myrtle Street, Liverpool L7 7DG, England.

J. H. Marshall, LRCP, MRCS, Orthopaedic Senior House Officer Arrowe Park Hospital, Arrowe Park Road, Upton, Wirral L49 5PE, England.

Correspondence to Mr M. P. Manning.

(C) 1987 British Editorial Society of Bone and Joint Surgery $0301-620 X / 87 / 1$ R $13 \$ 2.00$ review of 2611 arthroscopies, a $1.4 \%$ incidence of haemarthrosis was seen (personal communication, Sherman et al. 1985), whilst Dick et al. (1978), in their review of 3714 arthroscopies, recorded no haemarthroses nor any other complication of vessel damage. The development of a false aneurysm after arthroscopy has not previously been described.

The inferior lateral geniculate artery, because of its course along the lateral joint line, is clearly at risk when exposing the lateral side of the knee. The vessel is probably damaged more often than is realised, but complete division would result in vessel retraction with little bleeding. A lateral injury to the vessel, unusual because of its small lumen could, however, lead to a false aneurysm. Fairbank and Jamieson (1951) reported such aneurysms in two patients after open lateral meniscectomy and demonstrated the vulnerability of the anterior part of the inferior lateral geniculate artery as it passes through extra-synovial fat at the level of the upper margin of the meniscus. Their patients presented with recurrent haemarthroses because the false aneurysm communicated with the knee joint and also had pulsatile swellings with a bruit. Our patient presented with a subcutaneous false aneurysm, probably caused by the sharp trocar used to make a path for the arthroscope.

The rarity of this complication would not lead us to advocate a different approach for arthroscopy but we would recommend that, if the complication is suspected, exploration should be performed forthwith.

We would like to express our thanks to $\mathrm{Mr} \mathrm{R}$. Johnson for allowing us to report his case.

\section{REFERENCES}

Dick W, Glinz W, Henche HR, et al. Komplikationen der Arthroskopie: eine Analyse von 3714 Fällen. Arch Orthop Trauma Surg 1978;92:69-73. (Eng. Abstr.)

Fairbank TJ, Jamieson ES. A complication of lateral meniscectomy. $J$ Bone Joint Surg [Br] 1951 ;33-B:567-70. 